\title{
Motion Vector Sharing and Bitrate Allocation for 3D Video-Plus-Depth Coding
}

\author{
Ismaël Daribo, Christophe Tillier, and Béatrice Pesquet-Popescu (EURASIP Member)
}

Signal and Image Processing Department, Telecom ParisTech, 46 Rue Barrault, Cedex 13, 75634 Paris, France

Correspondence should be addressed to Béatrice Pesquet-Popescu, pesquet@tsi.enst.fr

Received 26 October 2007; Revised 14 March 2008; Accepted 21 May 2008

Recommended by A. Enis Çetin

\begin{abstract}
The video-plus-depth data representation uses a regular texture video enriched with the so-called depth map, providing the depth distance for each pixel. The compression efficiency is usually higher for smooth, gray level data representing the depth map than for classical video texture. However, improvements of the coding efficiency are still possible, taking into account the fact that the video and the depth map sequences are strongly correlated. Classically, the correlation between the texture motion vectors and the depth map motion vectors is not exploited in the coding process. The aim of this paper is to reduce the amount of information for describing the motion of the texture video and of the depth map sequences by sharing one common motion vector field. Furthermore, in the literature, the bitrate control scheme generally fixes for the depth map sequence a percentage of $20 \%$ of the texture stream bitrate. However, this fixed percentage can affect the depth coding efficiency, and it should also depend on the content of each sequence. We propose a new bitrate allocation strategy between the texture and its associated per-pixel depth information. We provide comparative analysis to measure the quality of the resulting $3 \mathrm{D}+t$ sequences.
\end{abstract}

Copyright (C) 2009 Ismaël Daribo et al. This is an open access article distributed under the Creative Commons Attribution License, which permits unrestricted use, distribution, and reproduction in any medium, provided the original work is properly cited.

\section{Introduction}

Three-dimensional television (3DTV), as the next revolution in visual technology, promises to bring to the customers a new generation of services. Enjoy three-dimensional entertainments without wearing special additional glasses, navigate freely around a sportive show, to name but a few of the new promising 3DTV applications. Another target fields can be expected, like digital cinema, IMAX theaters, medicine, dentistry, air traffic control, military technologies, computer games, and so on. In the meantime, the development of digital TV and autostereoscopic displays allows to easily introduce 3D in broadcast applications like television. The creation and the transmission of autostereoscopic content has to be thought with the broadcast constraints, and especially with two of them: the adaptivity with respect to the different receiver capabilities (size, number of views, depth perception, etc.) and the backward compatibility allowing to extract the $2 \mathrm{D}$ information for existing $2 \mathrm{D}$ displays.

Among the various studies [1-6], recent researches give much attention to 3DTV [7], more specifically to depth image-based rendering (DIBR) approaches. Indeed, DIBR technique has been recognized as a promising tool which can synthesize some new "virtual" views from the so-called video-plus-depth data representation, instead of using the former 3DTV proposals, such as 3D models or stereoscopic images. The video-plus-depth data representation uses a regular color video enriched with the depth map providing the $Z$-distance for each pixel (Figure 1). This format is currently standardized by the motion pictures experts group (MPEG) within the MPEG-C part 3 framework [8] of the compression of the per pixel depth information within a conventional MPEG-2 transport stream.

In contrast to the conventional end-to-end stereoscopic video chain, where two monoscopic video streams, one for the left and one for the right eye, need to be encoded and transmitted, only one monoscopic video stream and an associated per pixel depth sequence need to be encoded within a video-plus-depth scheme. Thereafter, it allows to create more than two views at the receiver side if needed, while the transmission is still done over the existing digital video broadcast (DVB) infrastructure. Furthermore, the characteristics of depth images, different from normal 


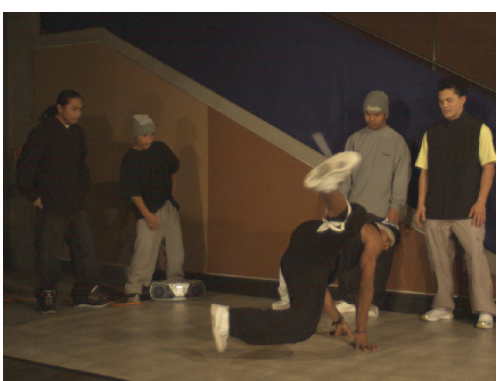

(a)

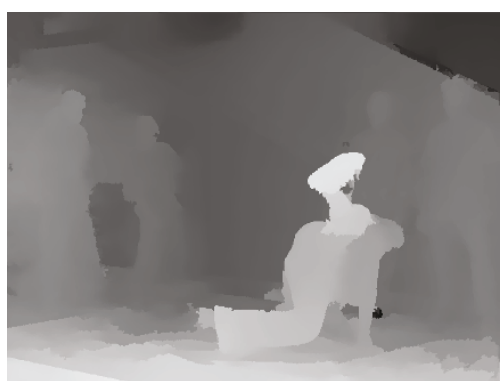

(b)

Figure 1: Example of texture image (a) and its associated depth image (b).

textured images, lead to a high-compression efficiency due to the smooth data representation, as illustrated in Figure 2.

For these advantages, the single view plus depth solution represents the most promising data representation format for the near future broadcast 3DTV system. An end-toend processing chain for such a system, starting with 3D acquisition, followed by postproduction, extracting depth information for 3D, rendering has been investigated by the European information society technologies (ISTs) project "advanced three-dimensional television system technologies" (ATTESTs) [9]. The ATTEST concept outlines different functional buildings blocks, as shown in Figure 3. A 3DTV signal is processed through a chain composed by different units: the $3 \mathrm{D}$ content generation, the $3 \mathrm{D}$ video coding, the transmission, the "virtual" view synthesis, and the display.

In this paper, an alternative method for encoding videoplus-depth sequences that utilizes a novel joint motion estimator is presented. Classically, the correlation between the texture motion vectors and the depth sequence motion vectors is not exploited in the coding process. One of the aims of this paper is to reduce the amount of information for describing the motion of the texture video and the depth map sequences by sharing one common motion vector field. Intuitively, the texture video and the depth map sequences have common characteristics, since they describe the same scene with the same point of view. For that reason, in both domains (color-surface structure and distance information) boundaries coincide and the direction of motion is the same. Our approach exploits the physical relation between the motion in both videos, the texture and depth map videos. However, the disadvantage is that it cannot handle scenarios containing motion in the $Z$ axis, which is not perceptible in the texture video, but is present in the depth map sequence. The correlation between the motion vectors between the texture video and the depth sequence has already been exploited in the literature. For example, in [10], the motion vectors found for the texture video have been shared to the depth map, without any modification. In [11], H.264 is used for depth map coding to reduce the motion estimation complexity of the depth map encoding by using the decoded texture motion information. This improves basic motion vector sharing idea with some additional modifications on the vectors. It requires some bits for motion vectors, but still it is claimed to be good especially in low bitrates. In our approach, the motion vector sharing idea is extended, by introducing into the estimation criterion the minimization of the two energies, of the texture video, and of the depth map.

Furthermore, in the literature, the bitrate control scheme generally fixes for the depth map sequence a percentage of $20 \%$ of the texture stream bitrate within MPEG-2 framework [12]. This value has been proposed, for example, in the project ATTEST. Considering a separable scheme where the texture is encoded independently with MPEG-2 (for backward compatibility with existing TV solutions) and the depth map with MPEG-4, this percentage can go down to 5-10\%. However, this fixed percentage can affect the depth coding efficiency, and this percentage should also depend of the specificities of each video. We propose a new bitrate allocation strategy, which considers both the texture and its associated per pixel depth information.

The remainder of this paper is structured as follows. In Section 2, we present the existing work on video-plusdepth format. The extensions of the video-plus-depth coding are described in Sections 3 and 4. Section 5 shows the experimental results. We finally summarize our work in Section 6.

\section{Video-Plus-Depth}

3DTV needs specific requirements like high quality, backward compatibility with current digital TV, interactivity, which can be used to support the autostereoscopic application scenarios. The high quality requirement supposes a large amount of data to be transmitted on the conventional 2D video channel. In addition, backward compatibility needs to allow the extraction of 2D information for the existing 2DTV displays. In the end, 3DTV applications need some kind of reactivity of the system in relation to user actions. Among all the potential 3D representation candidates (3D models, light field, ray space, plane sweep, etc.), video-plus-depth framework is the most suitable representation for an endto-end broadcast 3DTV system in order to fulfill the above mentioned constraints.

Initially studied in computer vision field, the videoplus-depth representation provides a texture video and its associated depth map sequence. The texture video provides 


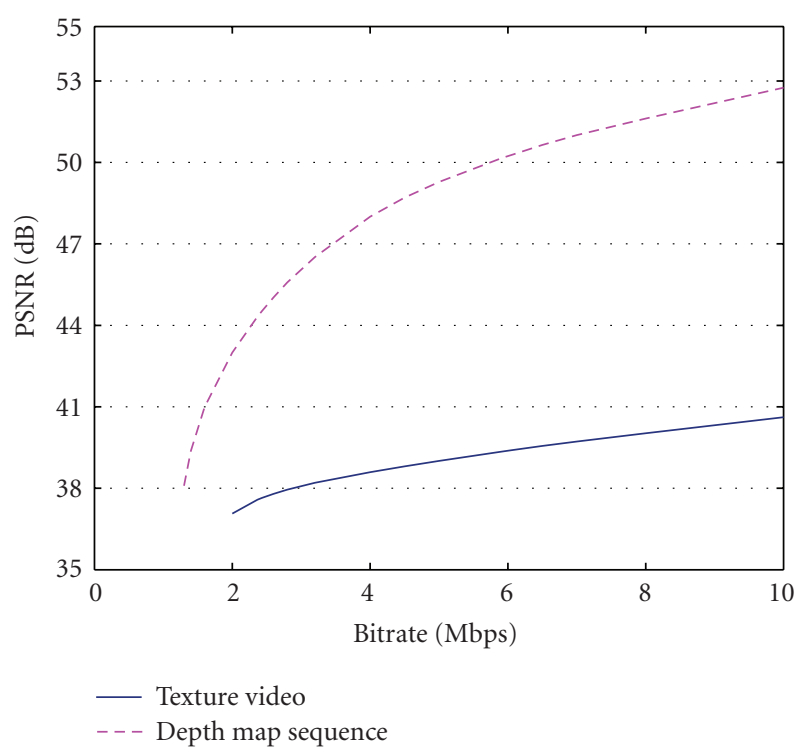

(a) Breakdancers cam0

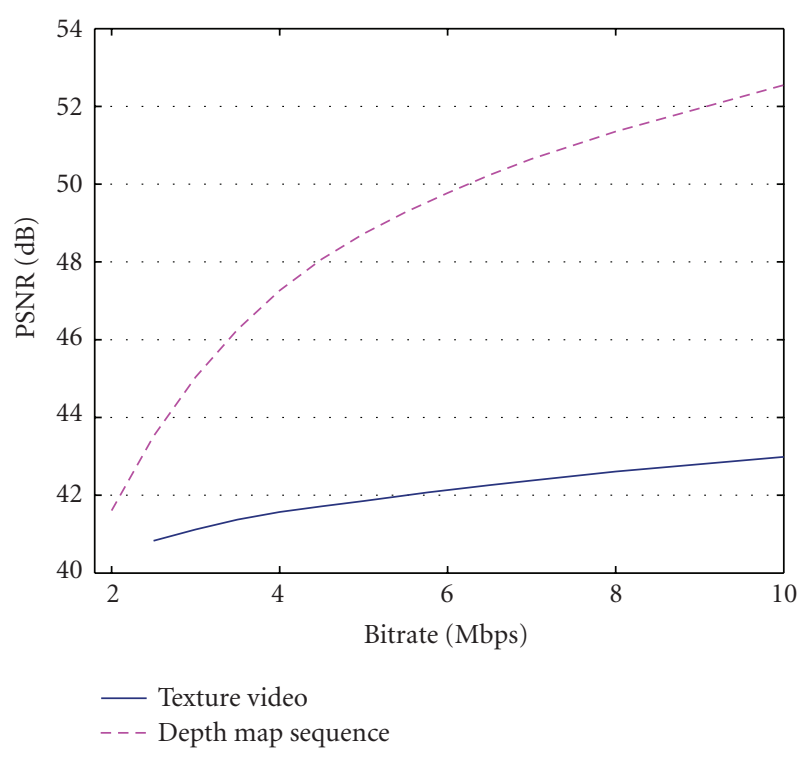

(b) Ballet cam0

FIGURE 2: Comparison of the compression efficiency between texture video and depth map sequence using the MPEG-2 reference software using a group of pictures (GOP) that consists of 12 frames with IBBP structure.

the surface, the color, the structure of the scene, whereas the depth map represents by means of a smoothed gray level representation the $Z$-distance between the optical center of the camera and a point in the visual scene.

Due to the very nature of the depth map picture, the smoothed gray level representation leads to a much higher compression efficiency than the texture video, as illustrated in Figure 2. Thus only a small extra bandwidth is needed for transmitting the depth map. Moreover, 3DTV based on depth map permits the synthesizing of new "virtual" views, utilizing depth map information, as if they were captured

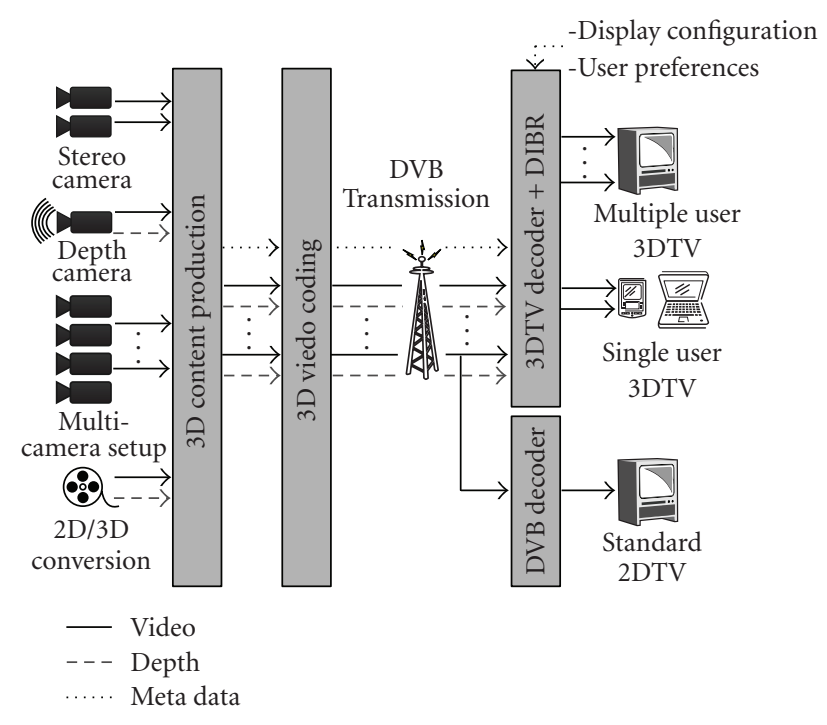

FIgure 3: The ATTEST 3DTV end-to-end system.

from a new "virtual" camera. Furthermore, this system is not optimized for a predefined screen size, and so, allows an easy customization of the depth effect.

MPEG has presented the MPEG-C Part 3 specification, which standardizes the video-plus-depth coding [8]. This specification is based on the encoding of $3 \mathrm{D}$ content inside a conventional MPEG-2 transport stream, which includes the texture video, the depth map sequences, and some auxiliary data. This standardized solution responds to the broadcast infrastructure needs. It provides interoperability of the content, display technology independence, capture technology independence, backward compatibility, compression efficiency, and user controlled global depth range.

2.1. Virtual View Synthesis. Considering the end-to-end system for 3DTV illustrated in Figure 3, at the receiver side the final 3D images are reconstructed by using DIBR, utilizing the transmitted reference view enriched with its associated per pixel depth information. This scheme, also called 3D image warping in the computer graphics literature [13], consists in first doing a projection from the $2 \mathrm{D}$ original camera image plane to the $3 \mathrm{D}$ coordinates. Thereafter, a second projection from the $3 \mathrm{D}$ coordinates is applied to the image plane of the desired virtual camera, using the respective depth values. Due to sharp horizontal changes in the depth map, the image warping reveals areas that are occluded in the reference view and become visible in some virtual views. To deal with this problem, averaging filters or more complex extrapolation techniques [12] are used to fill these occlusions.

We can distinguish two roles for the transmitted reference video stream. One is to consider it as a center view, and so a viewpoint translation and rotation on it will result in the virtual left and right views. Another configuration considers the transmitted real view as the right or left view. So, instead of generating two virtual views at the receiver side, just one is needed to reconstruct a stereoscopic scheme 


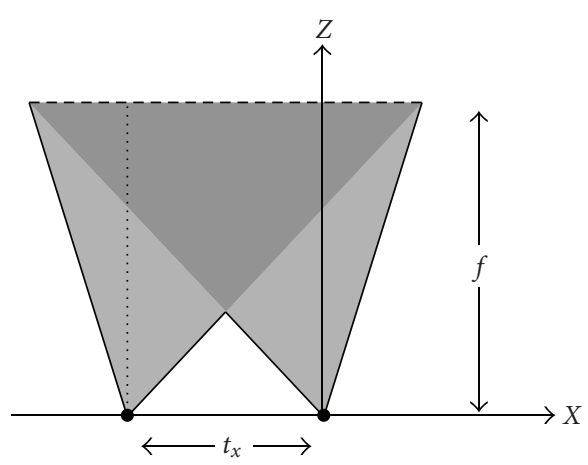

FIgURE 4: Shift-sensor camera setup: $t_{x}$ is the distance between cameras, $f$ is the focal length of the reference camera, and $Z$ represents the depth axis.

together with the depth information. In the sequel of this paper, we will consider that we only transmit the right view. Of course, this approach has some limitations: the virtual left view is generated from a double longer translation, causing more and bigger newly exposed areas. However, the quality of the right view is not at all affected. Consequently, the binocular perception is better supported and the depth sensation is better appreciated with an asymmetric quality than a reduction of quality in both views, as experimented in $[14,15]$.

Considering a system of parallel camera configuration (with known parameters) to generate stereoscopic content from the so-called shift-sensor approach (Figure 4), the warped view is performed by a projection, a horizontal translation, and a reprojection of pixels. The transformation that defines the new coordinates in the virtual view $\left(x_{\text {virt }}, y\right)$ from the reference view at $\left(x_{\text {ref }}, y\right)$ according to depth value $Z$ is calculated as

$$
x_{\mathrm{virt}}=x_{\mathrm{ref}}+\frac{t_{x} \times f}{Z},
$$

where $t_{x}$ is the distance between the reference camera and the virtual camera (commonly equal to the average human eyes separation), and $f$ is the focal length of the reference camera. In this case, a pixel and the associated warped pixel have the same vertical coordinates due to the chosen camera configuration.

Preprocessing the depth map allows to reduce the number and the size of holes created by the warping [16]. Nevertheless, some holes can remain, requiring a last step of hole filling, consisting in an interpolation of the missing values [17].

\section{Motion Prediction}

High compression efficiency is achieved by using motion estimation and compensation. Temporal redundancies are removed by estimating the motion between frames in the sequence and then generating the motion vector field, which minimizes the temporal prediction error. The motion vectors (MVs) for temporal prediction reside in the predictive $\mathrm{P}$ frames and the bidirectional B frames. Consequently, in a
TABLE 1: Percentage of the energy of the motion field vector inside the interview sequence.

\begin{tabular}{lcc}
\hline & Static object & Motion object \\
\hline Texture & $38.87 \%$ & $61.13 \%$ \\
Depth map & $20.01 \%$ & $79.99 \%$ \\
\hline
\end{tabular}

TABLe 2: Comparison of the mean value of the correlation coefficient and the difference value between all the MV and the MV belonging to the objects in movement.

\begin{tabular}{lcccc}
\hline & Correlation & $\begin{array}{c}\text { Correlation } \\
\text { with mask }\end{array}$ & Difference & $\begin{array}{c}\text { Difference } \\
\text { with mask }\end{array}$ \\
\hline $\begin{array}{l}\text { Horizontal } \\
\text { component }\end{array}$ & 0.2003 & 0.2675 & 0.3657 & 0.1790 \\
$\begin{array}{l}\text { Vertical } \\
\text { component }\end{array}$ & 0.1196 & 0.1679 & 0.3387 & 0.1146 \\
\hline
\end{tabular}

typical GOP for broadcasting purposes having the structure IBBPBBPBBPBB, the number of coded macroblocks in temporal predictive mode can reach $40 \%$ of the total number macroblock at low bitrate (as shown in Figure 5), and as a result, the transmission of motion data consumes a large part of the bitstream for low-bitrate coders.

The video-plus-depth stream contains usually twice this number of motion vector fields, respectively, for the texture and for the depth temporal prediction. Instead of working on the efficiency of the two motion vectors, in order to minimize the prediction error in both cases, we show that only one motion vector field can be transmitted inside the global stream, since the motion in both videos is correlated.

3.1. Motion Correlation. As the texture video and the depth map are spatially correlated, the motion vectors in the two sequences should also be correlated.

To prove this hypothesis, one experiment has been performed. The observation of the motion vectors confirms the correlated location of the motion information. Indeed in Figure 6, the similarities of objects boundaries in the texture and in the depth map are highlighted. Actually, the two videos describe the same scene with the same point of view. Consequently, the motion contained in the two sequences is similar at the same spatial location, and takes the similar directions (Figure 8). As expected, the motion analysis, the correlation coefficient, and the average difference between the MV shown in Figure 9 confirm the correlation between the MV.

Moreover, a second experiment is performed only on the MV of the object in movement by means of the associated segmentation mask sequence (Figure 10). The mask sequence allows to easily identifies the different layered objects at different depth levels. Indeed, Table 1 confirms that the energy of the MV of the characters are more important. As shown in the Figure 11, the correlation between the MV of the texture and the MV of the depth map gains a small amelioration in the correlation coefficient and a reduction in the average difference value as shown in Table 2. 


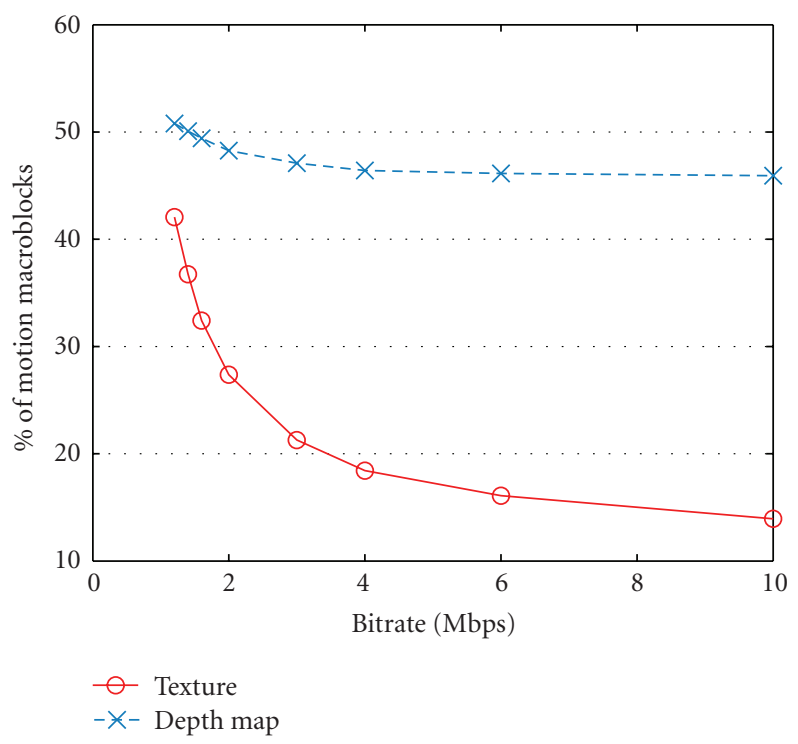

(a) Ballet sequence

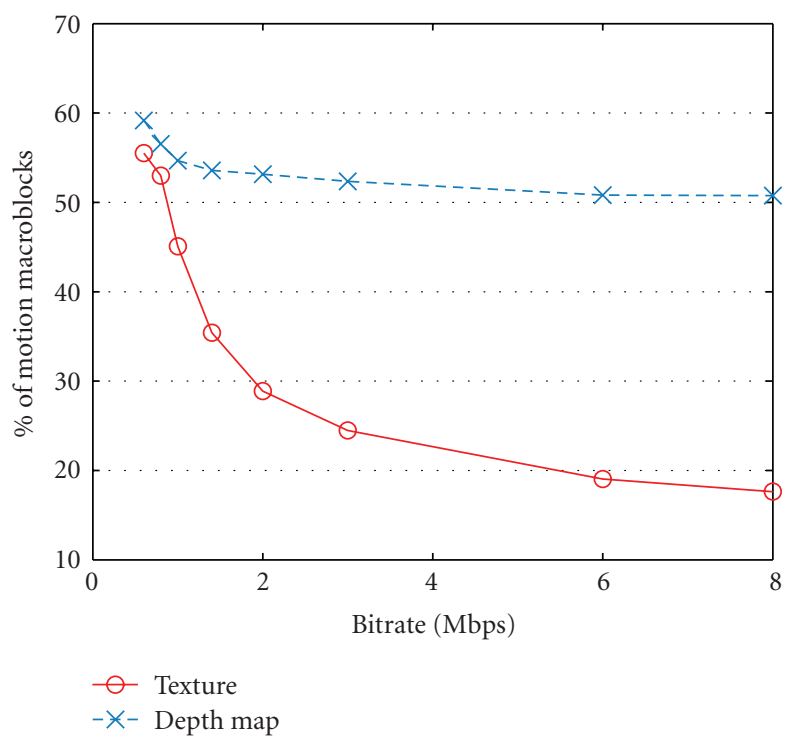

(c) Interview sequence

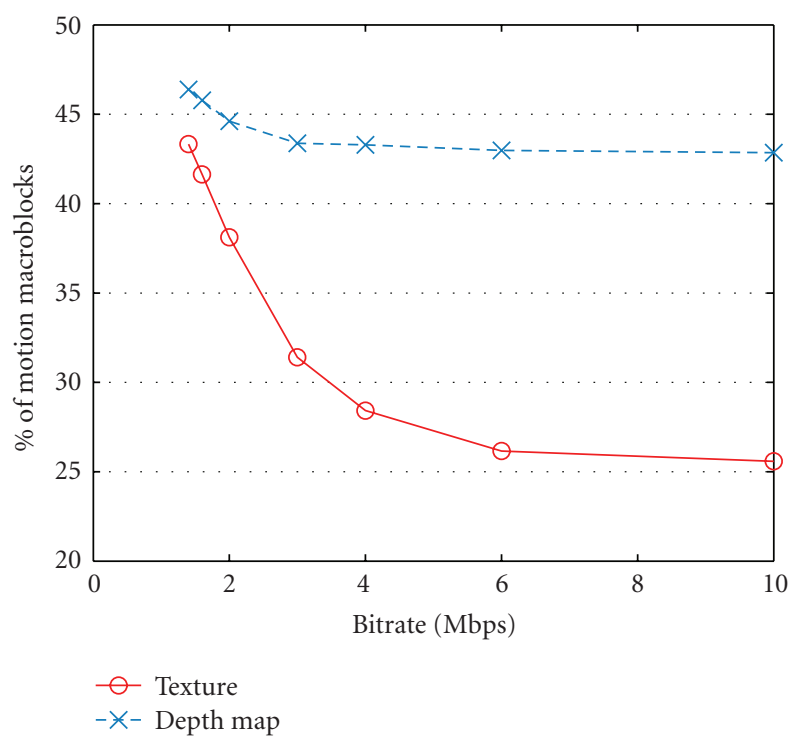

(b) Breakdancers sequence

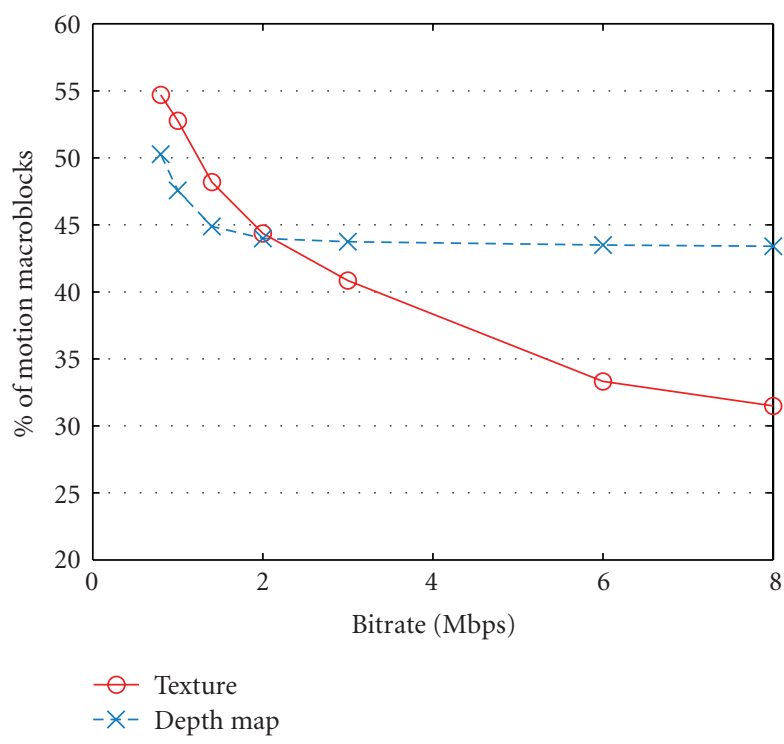

(d) Orbi sequence

FIGURE 5: Percentage of the coded predictive (forward and backward) macroblocks inside the video sequence.

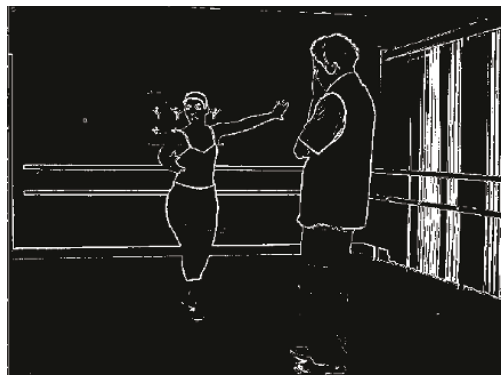

(a)

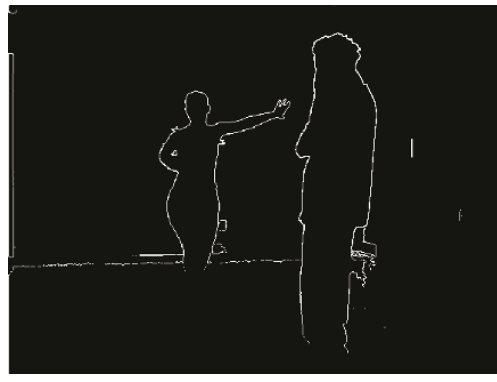

(b)

FIGURE 6: Edges in the texture image (left) and the associated depth image edges (right) from the sequence Ballet. 


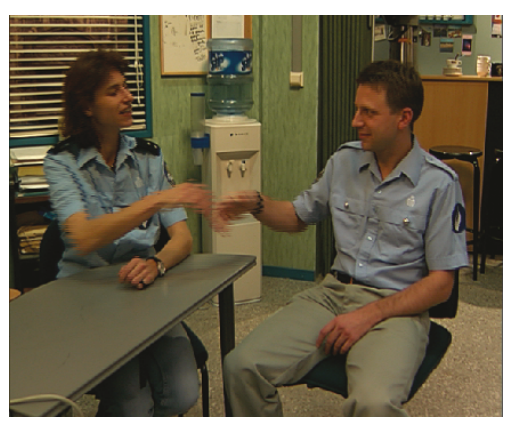

(a)

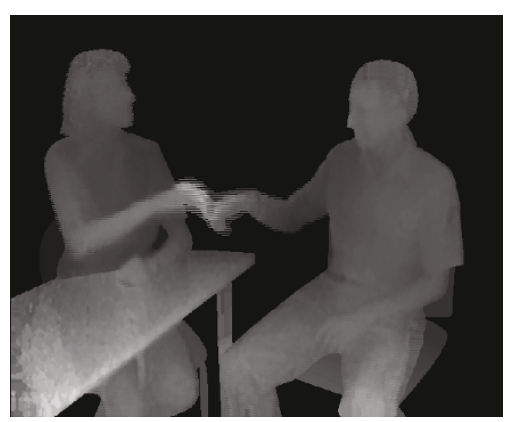

(b)

FIGURE 7: Example of texture image (a) and its associated depth image (b) from the frame 109 of the sequence interview. The two policemen are shaking hands which yields a lot of motion vectors.

3.2. Joint Motion Estimation. Among the various techniques for motion estimation (ME), block matching has been adopted in all international standards for video coding due to its simplicity and effectiveness. In this method, each frame is partitioned in nonoverlapping blocks of pixels, and each block is predicted from a block of equal size in the reference frame. The MV of a block is estimated by considering the best matching block, corresponding in general to the minimum mean square error (MSE) or mean absolute error (MAE) [18] with respect to the previous frame. Let $F^{t}(x, y)$ denote the image intensity of the $t$ th frame at the spatial location $(x, y)$. The vector $\left(v_{x}, v_{y}\right)$ maps points in the current frame $F^{t+1}$ to their corresponding locations in the previous frame $F^{t}$. For illustration, MSE is defined as follows:

$$
\text { MSE }=\frac{1}{N^{2}} \sum_{x=0}^{N} \sum_{y=0}^{N}\left(F^{t+1}(x, y)-F^{t}\left(x+v_{x}, y+v_{y}\right)\right)^{2} .
$$

In Section 2, we argued about the need to share the MV by encoding and transmitting only one motion field for both the texture and depth videos. That leads to account for both the distortion in texture and depth map videos by defining a new motion estimation, where the distortion criterion to minimize is this time defined jointly for the video texture and the depth map as follows:

$$
\mathrm{MSE}_{j o i n t}=\alpha \mathrm{MSE}_{\text {depth }}+(1-\alpha) \mathrm{MSE}_{\text {texture }},
$$

where $\alpha \in[0,1]$ controls the relative importance given to the depth and to the texture for this estimation procedure. According to the proposed distortion metric, the resulting MV field is used for the two streams, and then encoded only once. The value $\alpha=0$ is a particular case already studied in [10], where only the MV from the texture information is considered to encode both the texture and the depth map sequences. In our method, we generalize this concept and investigate the problem of estimating a motion field which can reduce the temporal correlation as well for the depth information as for the texture data, by means of the joint estimation criterion. In the experiments, we tune the parameter $\alpha$ to find the optimal value depending on the content of the sequence.
3.3. Motion Sharing. Once the common MV is found, it has to be encoded for transmission. The motion field used to encode both the texture and the depth map sequences is placed in the texture bitstream, to ensure the required backward compatibility with current TV set-top boxes.

As illustrated in Figure 12, the MVs are shared and only sent once in the global video-plus-depth stream. Consequently, this strategy allows more bandwidth resources to the depth map residues. Moreover, it overcomes the imperfect match between the two MV fields. In fact, the correlation error is less significant compared with the gain in bandwidth.

\section{Content Aware Bitrate Allocation}

In this section, we consider the problem of finding a ratedistortion allocation strategy, which may jointly optimize the resulting video quality and the required bitrate sharing between the texture and depth map data.

To this end, for each GOP the bits are allocated taking into account the ratio of the variances of the pictures in the texture video and the depth map sequence. For the $\mathrm{P}$ and the $\mathrm{B}$ frames, this variance is computed on the displaced frame difference (DFD), defined as

$$
\Delta F^{t}(x, y)=F^{t+1}(x, y)-F^{t}\left(x+v_{x}, y+v_{y}\right)
$$

with $\left(v_{x}, v_{y}\right)$ being the MV which minimizes the MSE measure defined in (3). The variance of this DFD is given by

$$
\sigma_{v_{x}, v_{y}}^{2}=\frac{1}{N^{2}} \sum_{x=0}^{N} \sum_{y=0}^{N}\left(\Delta F_{v_{x}, v_{y}}^{t}(x, y)-\overline{\Delta F_{v_{x}, v_{y}}^{t}}\right)^{2},
$$

where $\overline{\Delta F_{v_{x}, v_{y}}^{t}}$ denotes its average value, that is,

$$
\overline{\Delta F_{v_{x}, v_{y}}^{t}}=\sum_{x=0}^{N} \sum_{y=0}^{N} \Delta F^{t}(x, y)
$$




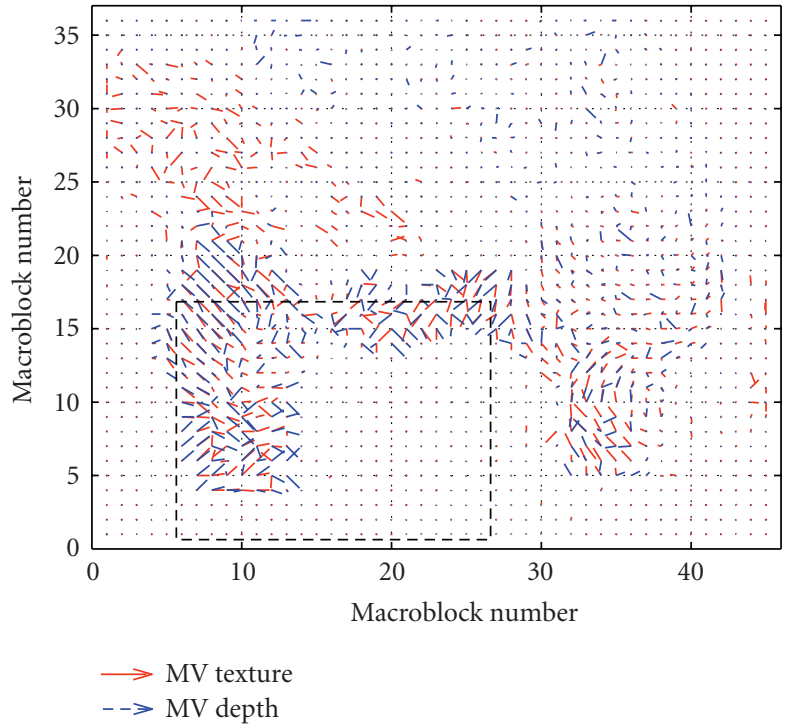

(a) Motion vector field

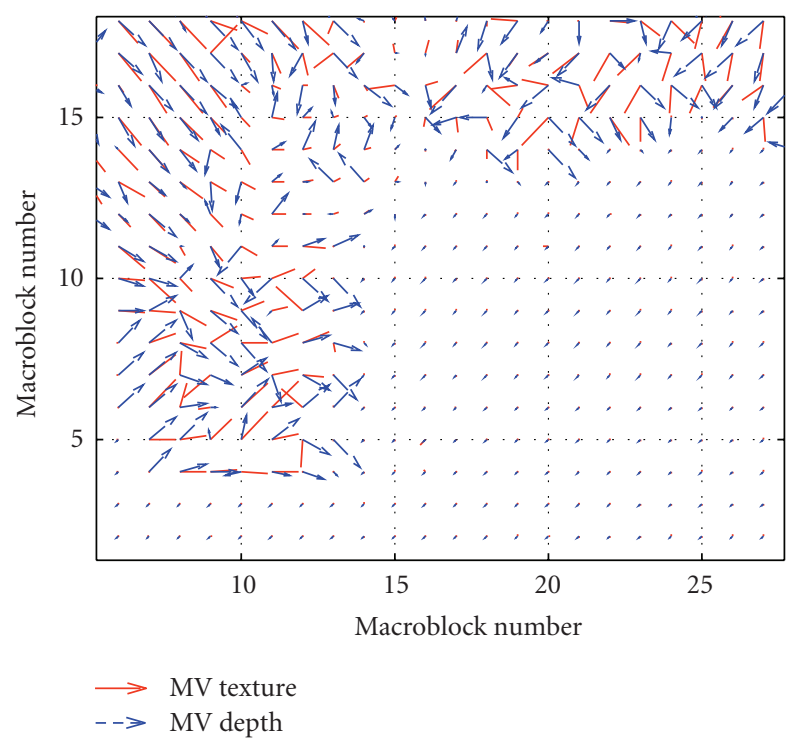

(b) Zoom on the field

Figure 8: Example of motion vector field from the frame 109 of the sequence interview (Figure 7).

4.1. Bit Allocation Strategy. Finding the optimal rate allocation between the texture and the depth map is a Lagrangian optimization problem, with a cost function $J$ involving the distortion $D$ weighted by the number of bits $R_{c}$ and $R_{d}$, respectively, associated with the texture and the depth map. By using a Lagrange multiplier $\lambda$ [19], this yields

$$
\min \{J\}, \quad \text { where } J=D+\lambda(R)
$$

where the Lagrangian parameter $\lambda>0$, if judiciously applied, can provide significant benefits.
Introducing the rate-distortion model at high resolution $D(R)[19]:$

$$
D(R)=a \sigma^{2} 2^{-2 R},
$$

where $a$ is a parameter depending on the distribution of the source, one can write the global distortion as

$$
D(R)=D_{c}+D_{d}=a_{d} \sigma_{d}^{2} 2^{-2 R_{d}}+a_{c} \sigma_{c}^{2} 2^{-2 R_{c}},
$$

where $a_{c}, a_{d}$ are constants associated with the distribution of the texture and depth map.

The needed bitrate to encode each stream is function of the global bitrate $R$ and the variance of the composing streams, texture, and depth map, as follows:

$$
\begin{aligned}
& R_{c}=\frac{R}{2}+\frac{1}{2} \log _{2} \frac{\sigma_{c}}{\sigma_{d}}, \\
& R_{d}=\frac{R}{2}+\frac{1}{2} \log _{2} \frac{\sigma_{d}}{\sigma_{c}},
\end{aligned}
$$

where $\sigma_{c}, \sigma_{d}$ are, respectively, the standard deviations of the texture and depth map.

With the variance of a frame defined in (5), we can estimate the average number of bits allocated for each stream composing the global video-plus-depth stream.

\section{Experimental Results and Discussion}

Our experiments evaluate the proposed motion estimation and bitrate allocation methods on two types of sequences providing a conventional video enriched with a depth map sequence. The first type contains two sequences: "Breakdanders" and "Ballet" $(1024 \times 768)$ at $15 \mathrm{fps}$ [20]. The depth maps of these sequences have been computed using a stereo matching algorithm. The second type contains the sequences "Interview" and "Orbi" $(720 \times 576)$ at $25 \mathrm{fps}$ [21], where the depth information is captured directly from the so-called $Z$ cam camera.

According to the MPEG-C Part3 specifications, and under constraints that the same encoder is used as well for the texture and the depth map, the experiments have been done with the MPEG-2 reference software. An IBBP GOP of 12 pictures was used for the configuration of the coder software.

One of the various MPEG2 industrial applications can be the storage on DVD support or the transmission over the digital broadcast using the DVB standard. The used bitrate has to satisfy at least the quality and the resolution of the picture for that an average viewer does not perceive any compression lossy data effect (compression artifacts, block effects, etc.). Firstly, in DVD case, considering an $\mathrm{SD}$ resolution $(720 \times 576)$ at $25 \mathrm{fps}$, the bitrate is between $4 \mathrm{Mbps}$ and $8 \mathrm{Mbps}$, that is, $0.39 \mathrm{bpp}$ and $0.77 \mathrm{bpp}$. Still in $\mathrm{SD}$ resolution, the digital television channels are transmitted using mostly a bitrate between $2 \mathrm{Mbps}$ and $8 \mathrm{Mbps}$, that is, $0.19 \mathrm{bpp}$ and $0.77 \mathrm{bpp}$ [22]. According to these values, the 


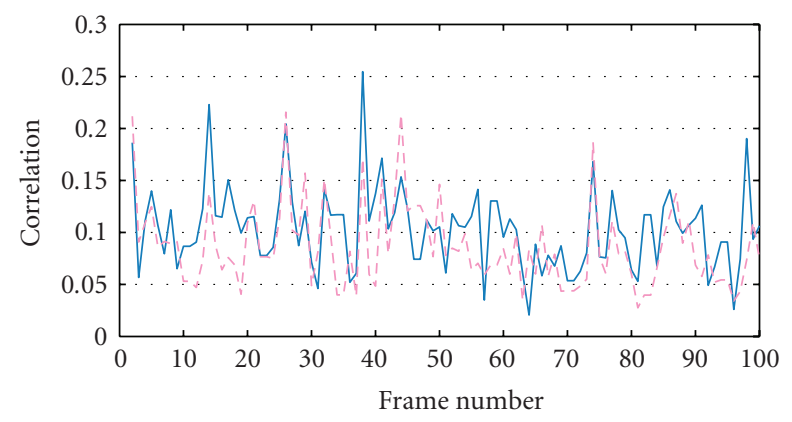

(a) Ballet-plus-depth correlation

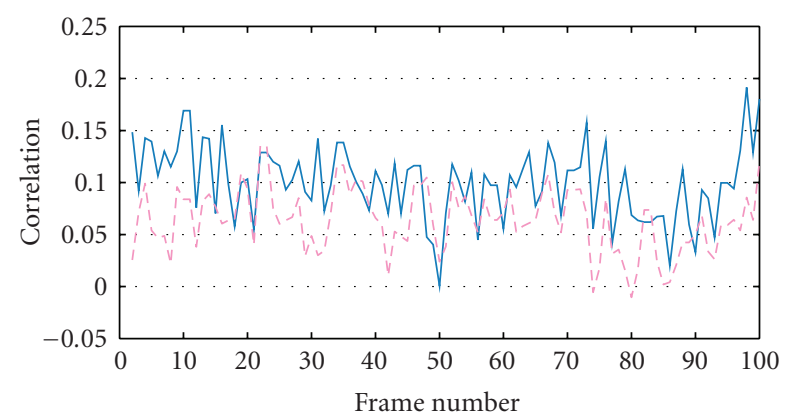

(c) Breakdancers-plus-depth correlation

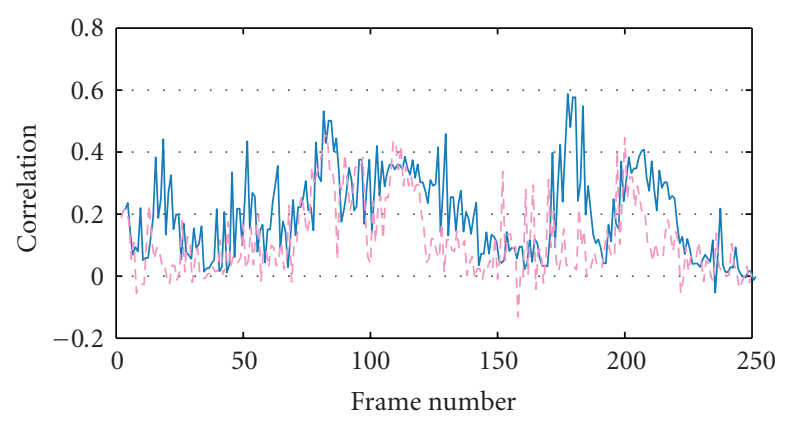

(e) Interview-plus-depth correlation

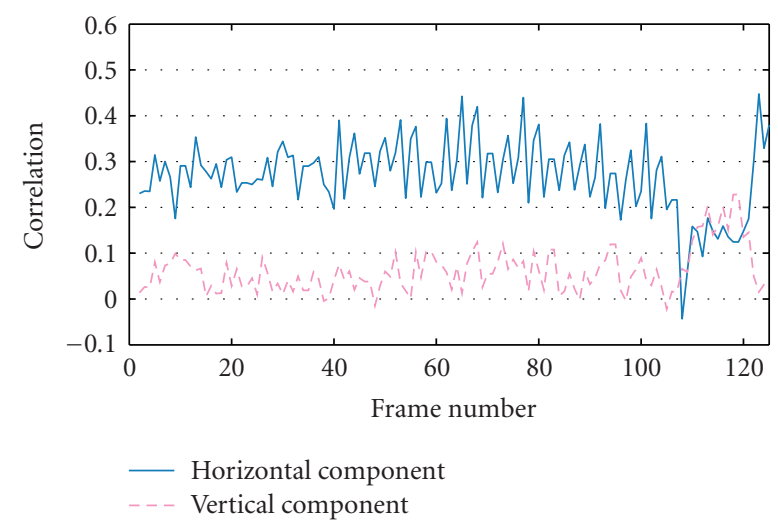

(g) Orbi-plus-depth correlation

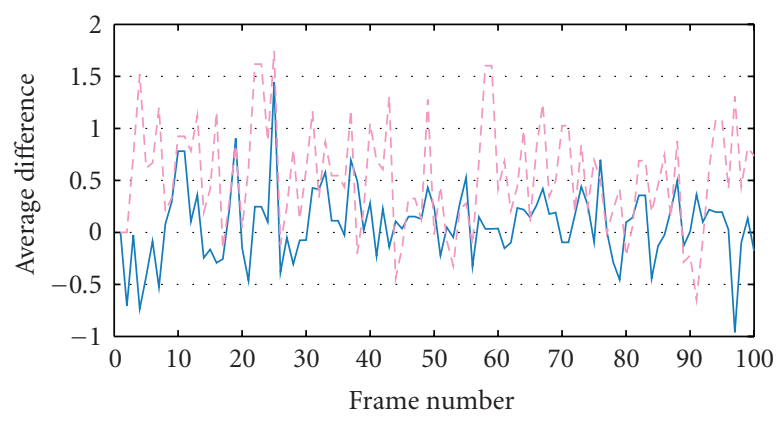

(b) Ballet-plus-depth average difference

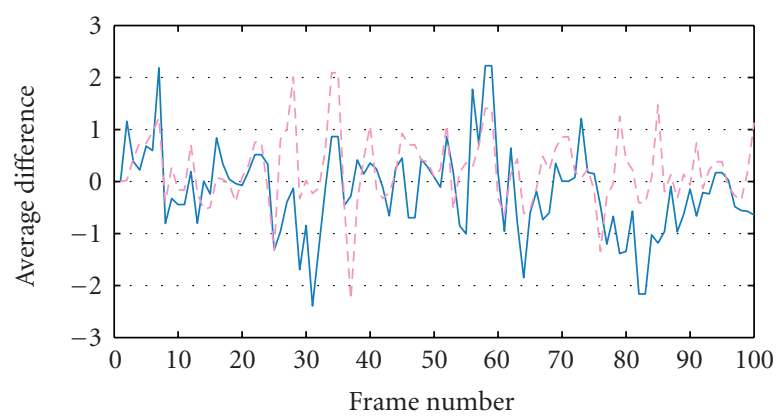

(d) Breakdancers-plus-depth average difference

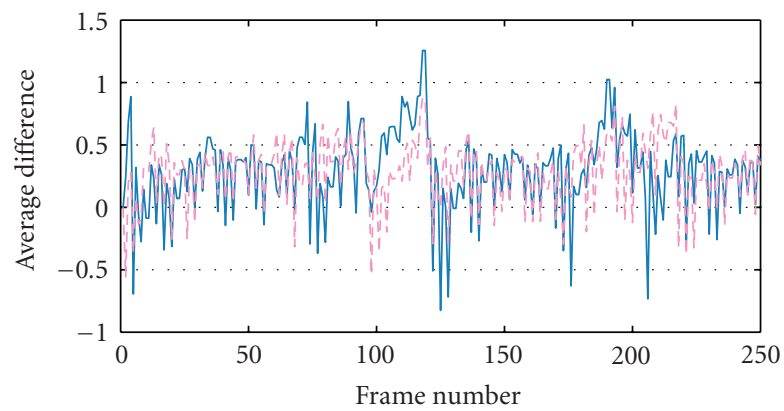

(f) Interview-plus-depth average difference

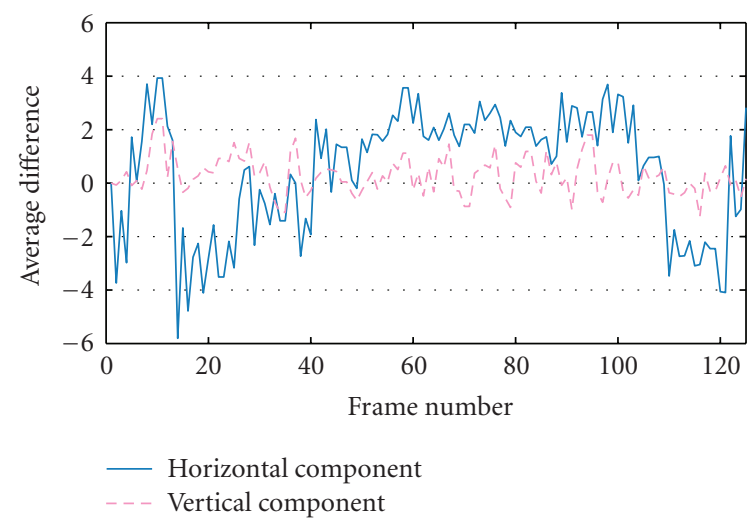

(h) Orbi-plus-depth average difference

FIGURE 9: Motion vector analysis: correlation and average difference between the MV of the texture and the MV of the depth map. 


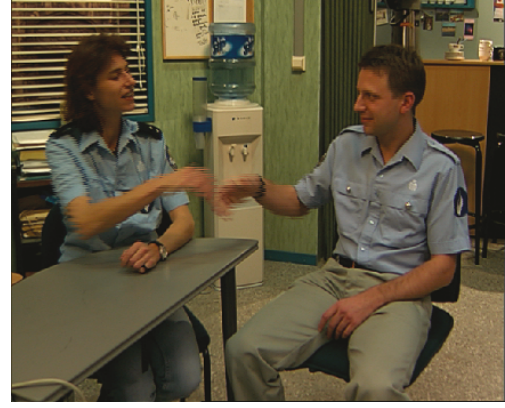

(a)

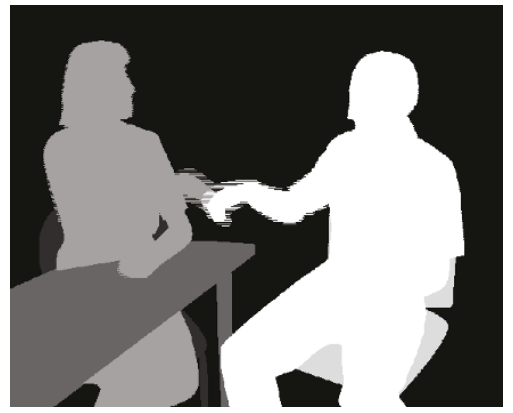

(b)

FIGURE 10: Example of texture image (a) and its associated mask image (b) from the frame 109 of the sequence interview. The two policemen are shaking hands which yields a lot of MV.

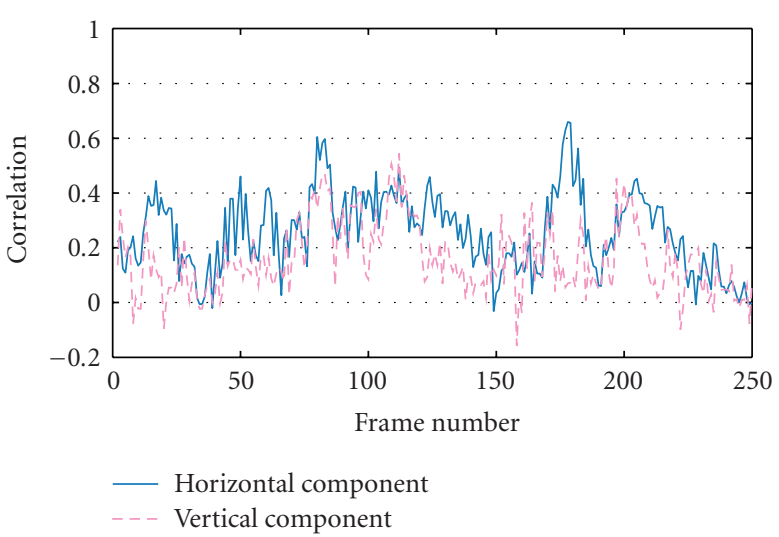

(a) Interview-plus-depth correlation

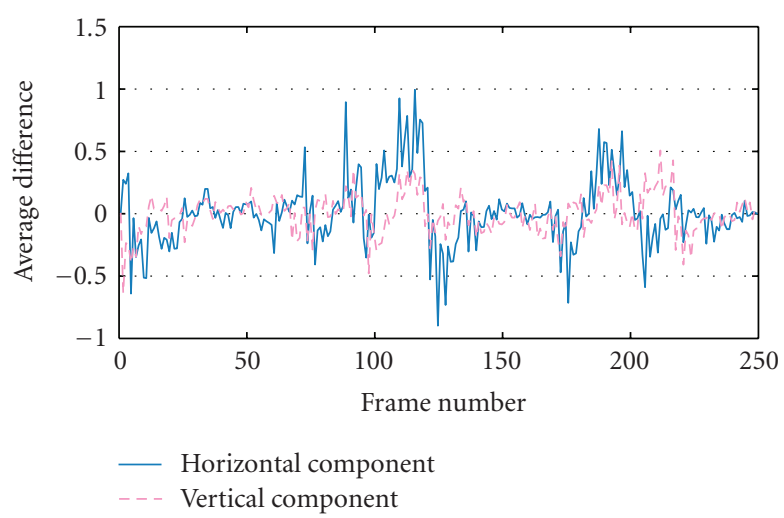

(b) Interview-plus-depth average difference

FIGURE 11: Motion vector analysis only on object in movement in the scene.

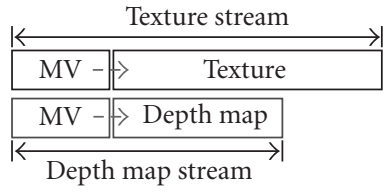

(a) Independent motion vector

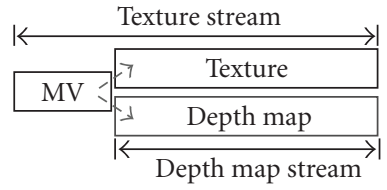

(b) Shared motion vector
FIGURE 12: Different strategies for MV encoding: (a) separate MV for texture and depth map and (b) a common MV field for texture and depth sequences.

test sequences are encoded, according to their own resolution and frame rate, in respect of the bitrate range used in digital content industry.

Figure 13 shows the PSNR of the texture video and of the depth map sequence when the parameter $\alpha$ varies between 0 and 1 . One can remark a sensible improvement of the depth map reconstruction (more than $1 \mathrm{~dB}$ ), for a small reduction in the texture video quality (between $0.4-0.8 \mathrm{~dB}$ ), when using the joint estimation criterion.
In order to find the optimal value of $\alpha$ for each test sequence, we tune the parameter and provide PSNR analysis of the reconstructed (virtual) sequence as illustrated in Figure 14 . The depth map bitrate is arbitrarily fixed to $20 \%$ to the texture bitrate. The curves highlight a value close to $\alpha=$ $0.2, \alpha=0.0$, and $\alpha=0.6$ as the best value for, respectively, the sequence "Ballet," "Breakdancers," and "Interview." This shows that estimating the MV only on the texture video does not lead to the best reconstruction of the virtual sequence, and the proposed trade off can largely improve the results.

As defined in (10), Table 3 shows for different sequences the variance ratio value which between texture video and depth map sequence for each type of frame in a GOP. Except for the "Breakdancers" sequence, the main variation in allocation affects the I frame. As a result, more bits are allocated to the texture stream than the depth map stream.

Considering the depth map bitrate equal to $20 \%$ of the texture bitrate, Figure 15 shows the resulting "virtual” PSNR. The joint motion estimation has been coupled with the new bitrate allocation. The results show better performance at high bitrate (between $0.5-1.5 \mathrm{~dB}$ ) for a small reduction at low bitrate (between 0.2-1 dB). 


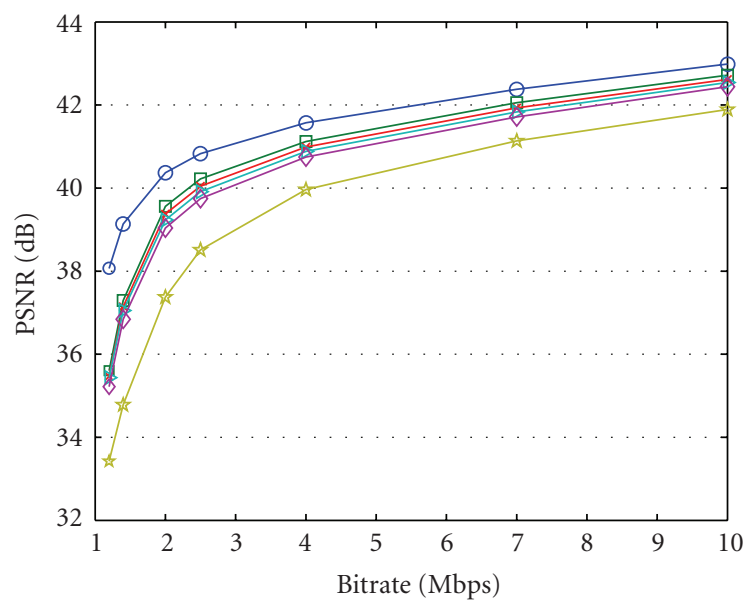

(a) Ballet texture

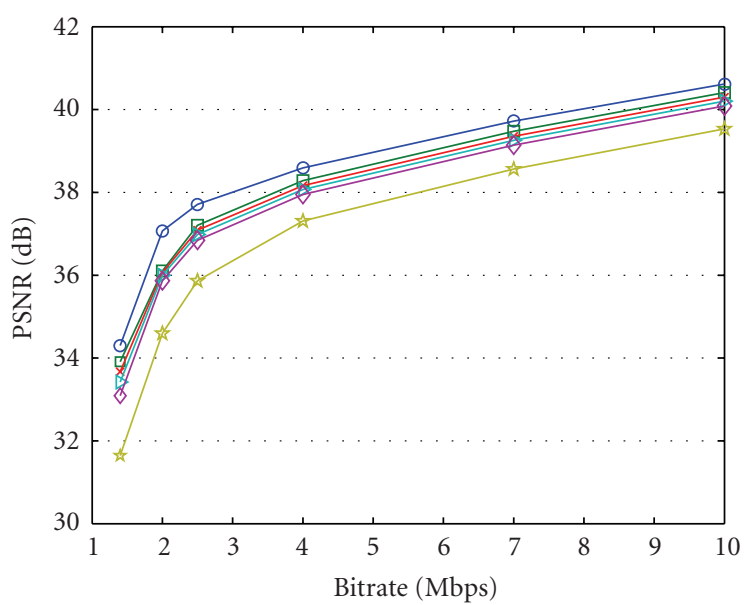

(c) Breakdancers texture

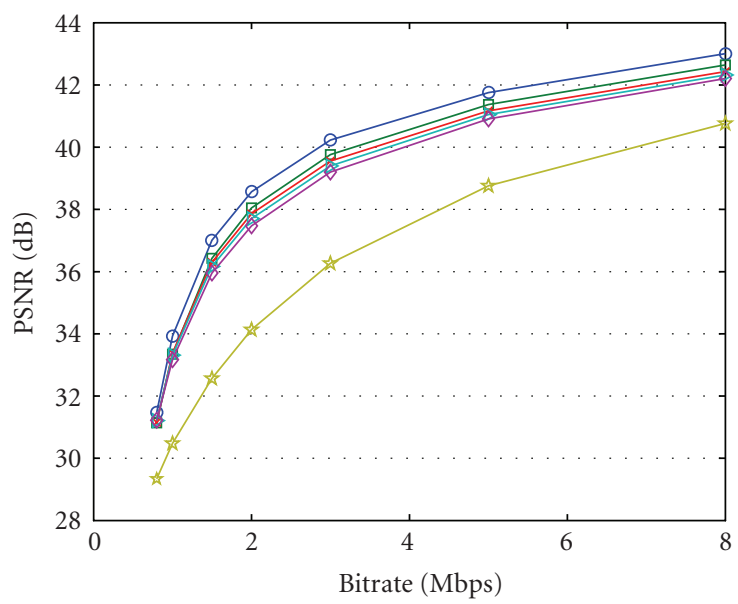

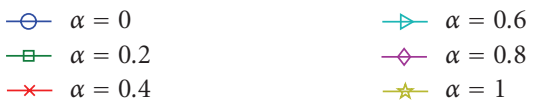

(e) Interview texture

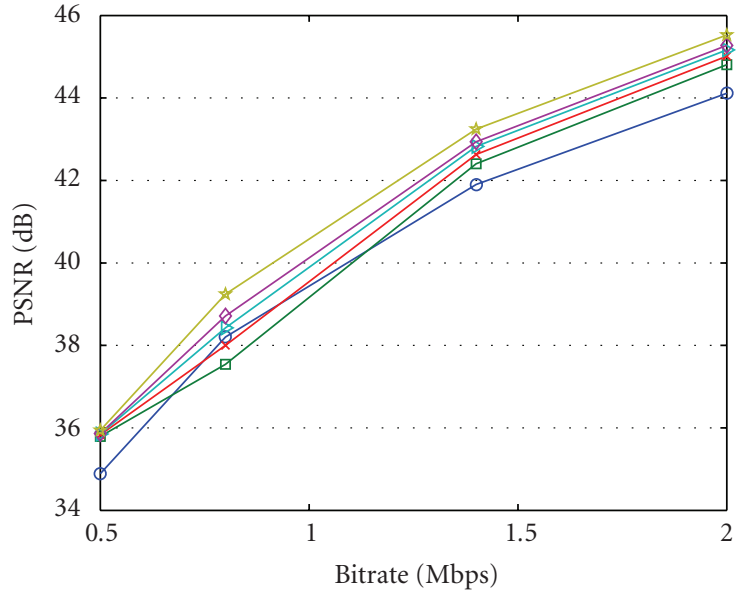

(b) Ballet depth map

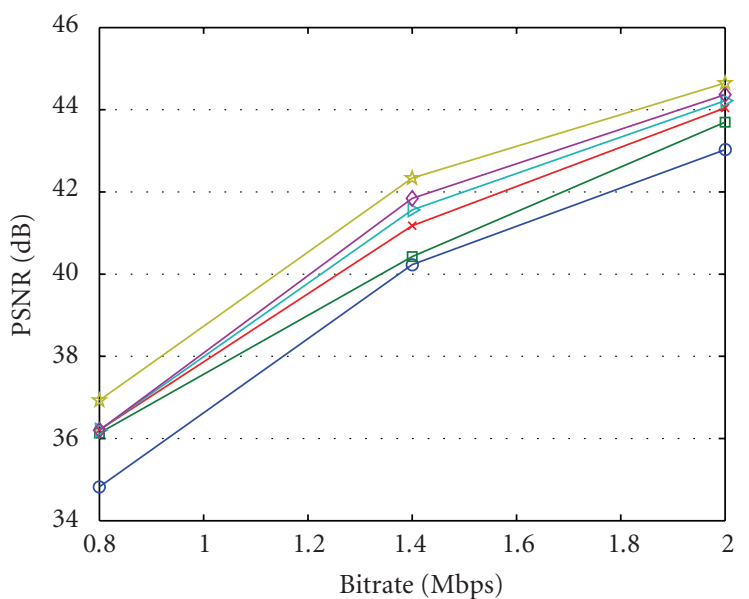

(d) Breakdancers depth map

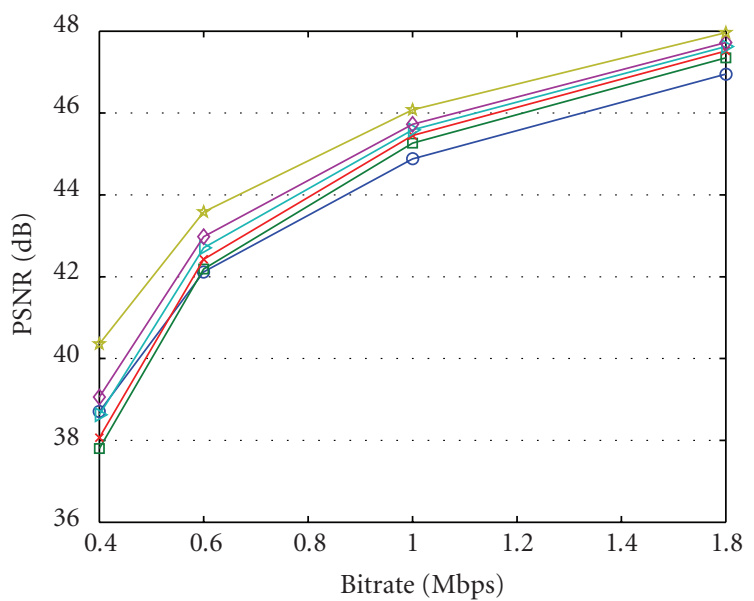

$$
\begin{aligned}
& -\alpha=0 \\
& \square \alpha=0.2 \\
& \star \alpha=0.4 \\
& \rightarrow \quad \alpha=0.6 \\
& \neg \quad \alpha=0.8 \\
& \text { 갓 } \alpha=1
\end{aligned}
$$

(f) Interview depth map

FIGURE 13: PSNR comparison with a joint MSE, for a variable parameter $\alpha \in[0,1]$. 


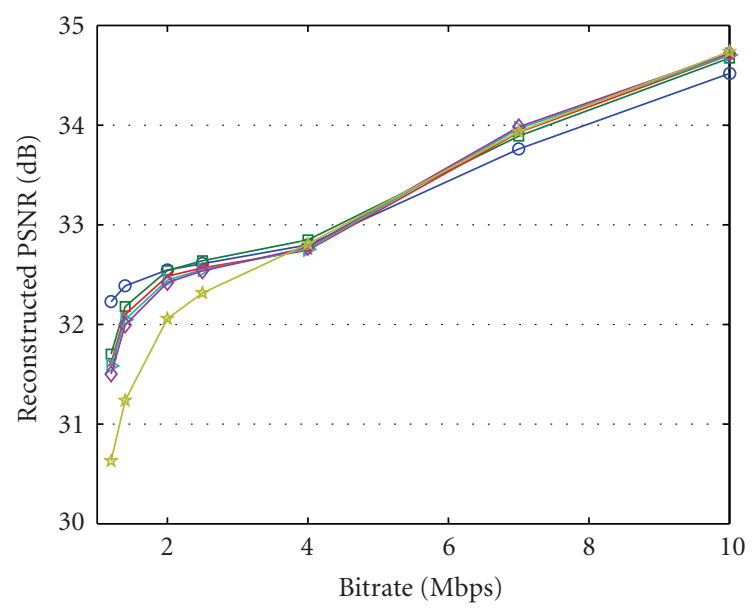

(a) "Virtual" Ballet sequence

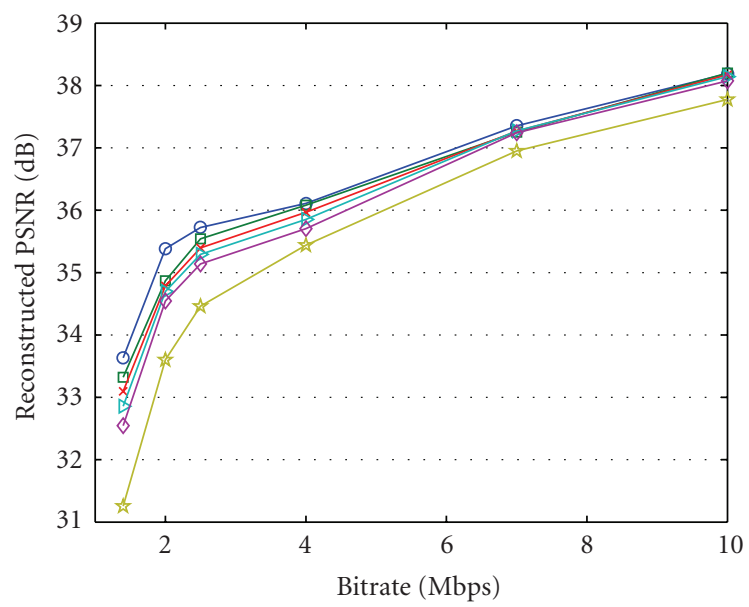

(b) "Virtual" Breakdancers sequence

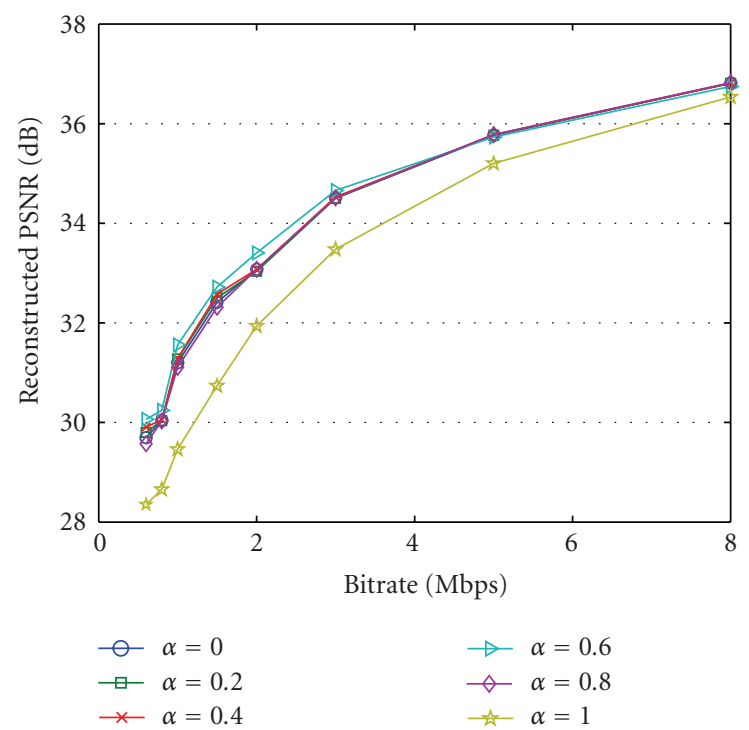

(c) "Virtual" Interview sequence

FIGURE 14: Search of the optimal $\alpha$ by comparison of the reconstructed "virtual" video PSNR. The depth map bitrate is equal to $20 \%$ of the texture bitrate.
TABle 3: Average variance ratio between the texture video and the depth map sequence.

\begin{tabular}{lccc}
\hline & \multicolumn{3}{c}{$\sigma_{c} / \sigma_{d}$} \\
\hline & $\mathrm{I}$ & $\mathrm{P}$ & $\mathrm{B}$ \\
Breakdancers & 0.3266 & 0.9414 & 1.0891 \\
Ballet & 0.6513 & 3.9866 & 4.3257 \\
Interview & 0.5646 & 1.0871 & 1.1159 \\
\hline
\end{tabular}

TABLe 4: Average MOS provided numerical indication of the perceived quality. MOS value is expressed between 1 and 5 , where 1 refers to lowest quality, and 5 to a highest quality.

\begin{tabular}{lcc}
\hline & Reference MOS & Proposed MOS \\
\hline Ballet (2.5 Mbps) & 2.7 & 2.4 \\
Ballet (7 Mbps) & 3.6 & 4.2 \\
Breakdancers (2.5 Mbps) & 2.8 & 3.4 \\
Breakdancers (7 Mbps) & 4.1 & 4.2 \\
Interview (1.5 Mbps) & 1.4 & 1.3 \\
Interview (5 Mbps) & 3.8 & $\mathbf{3 . 9}$ \\
\hline
\end{tabular}

Since 3D perception depends heavily on the stereoscopic vision of two sequences, the transmitted texture video and the reconstructed "virtual" sequence, it is difficult to evaluate the $3 \mathrm{D}$ perceived quality only by means of an objective evaluation model like the PSNR. Thus an additional validation is proposed through an subjective evaluation. For this, the perceived quality and the depth perception are conducted using the double stimulus continuous quality scale method (DSCQS) test methodology [23]. Nonexperts and inexperienced accessors are providing their opinion of the video quality and the video depth perception. The experiment gathers 15 accessors using the autostereoscopic Sharp LL-151-3D LCD Monitor. Average mean opinion score (MOS), according to the ITU-R Recommendation BT.50010 , is given in Table 4 . The results obtained confirms the objective results. It is shown an overall amelioration of the proposed method compare to the conventional MPEG2 bitrate allocation with an advantageous amelioration in high bitrate.

\section{Conclusion and Future Work}

This paper presented a novel method for the coding the video-plus-depth data by means of a joint estimation of the MV field for the texture motion information and the depth map sequence. Furthermore, we developed a bitrate allocation strategy between the texture and depth map stream based on a rate-distortion criterion. According to the MPEG-C Part 3 specifications, the texture video was encoded in an MPEG-2 stream for backward-compatibility purposes.

In future work, we aim at developing a new model for the rate-distortion optimization, which can take into account the quality of the reconstructed virtual view and also building a global distortion based on subjective quality. 


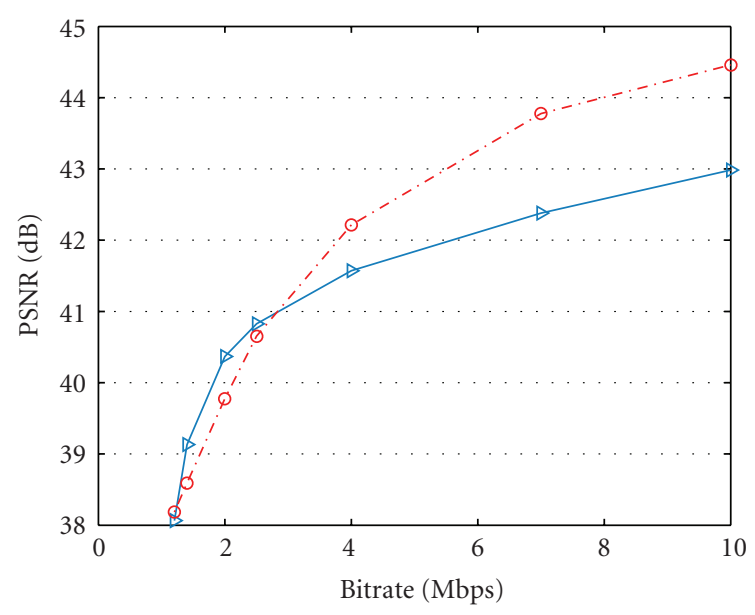

(a) "Transmitted" Ballet sequence

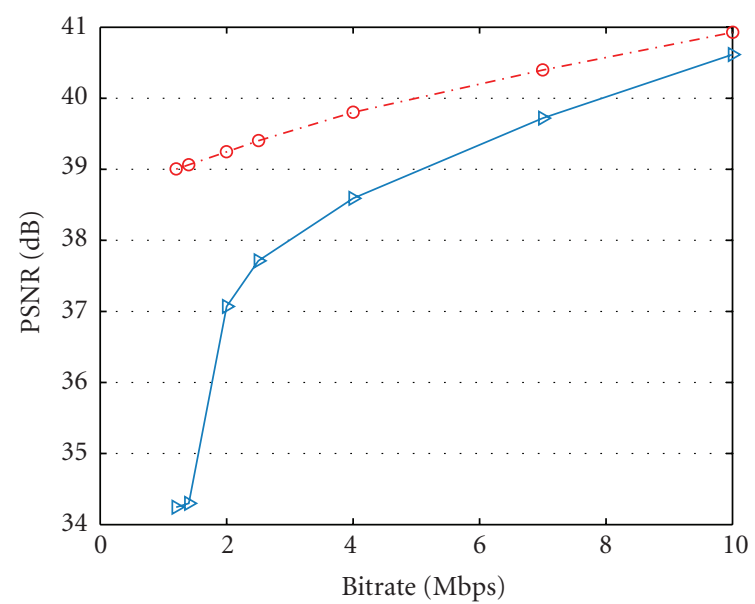

(c) "Transmitted" Breakdancers sequence

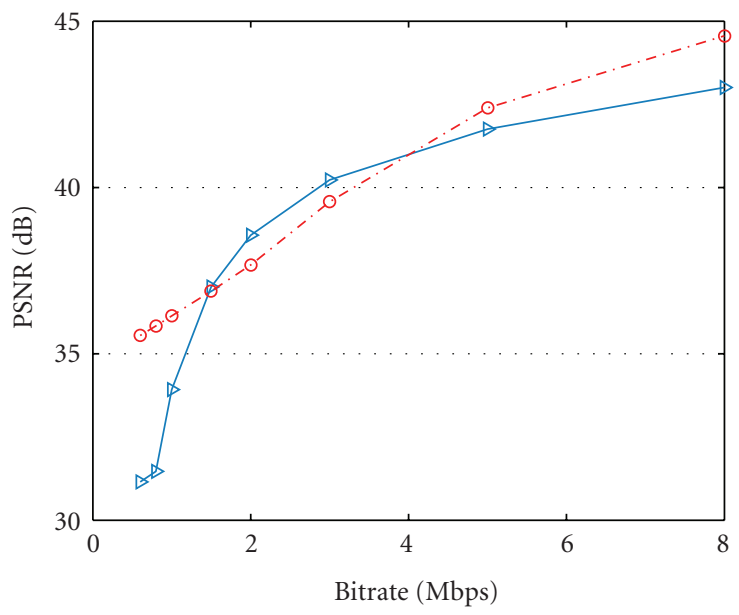

$\triangleright$ Reference

- - Proposed

(e) “Transmitted" Interview sequence

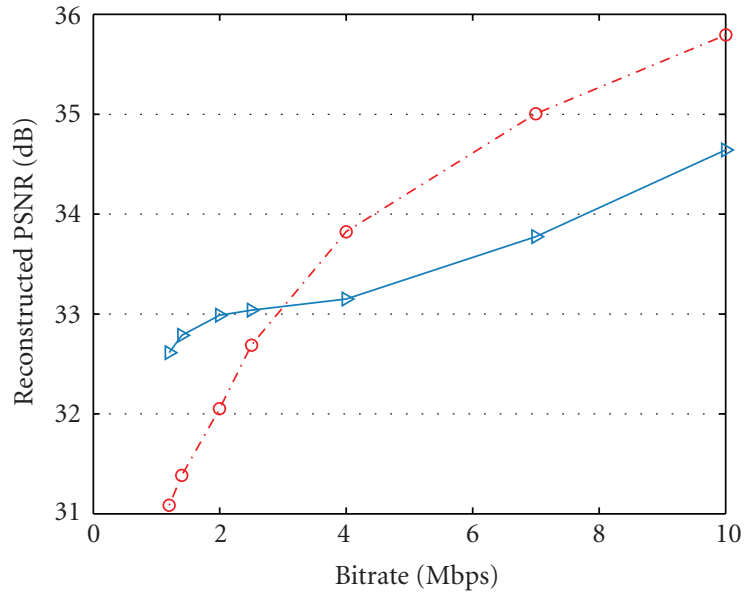

(b) "Virtual" Ballet sequence

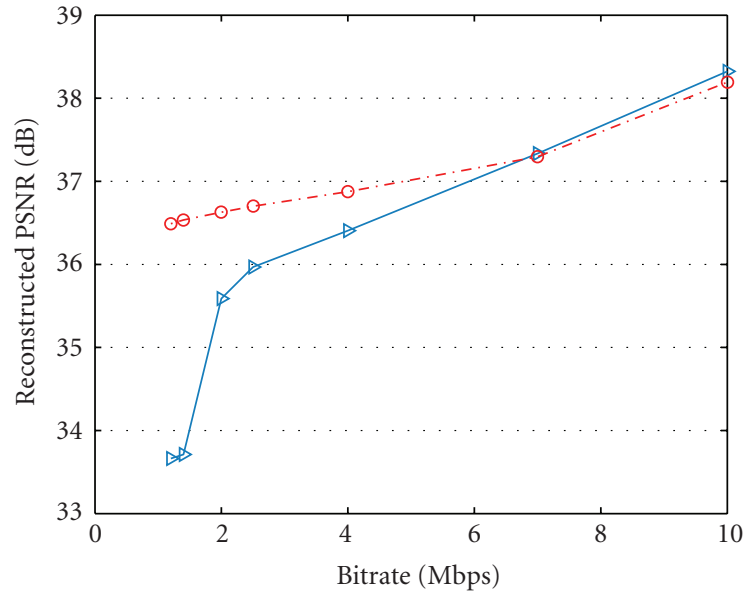

(d) "Virtual" Breakdancers sequence

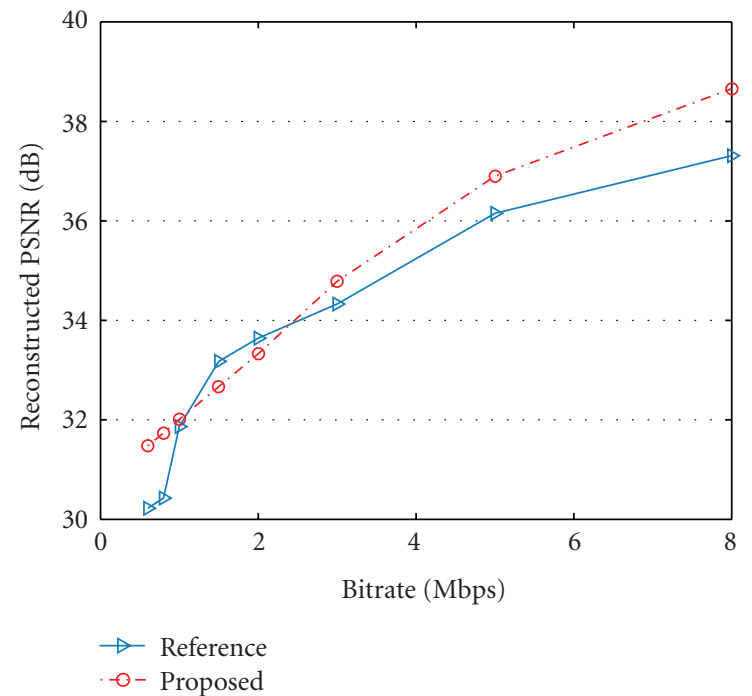

(f) "Virtual" Interview sequence

FIGURE 15: Resulting reconstructed PSNR of the "virtual" video using the new bitrate allocation with the PSNR of the other stereo view. Depth map bitrate equals $20 \%$ of the texture bitrate. 


\section{References}

[1] K. Yamamoto, M. Kitahara, H. Kimata, et al., "Multiview video coding using view interpolation and color correction," IEEE Transactions on Circuits and Systems for Video Technology, vol. 17, no. 11, pp. 1436-1449, 2007.

[2] S.-U. Yoon and Y.-S. Ho, "Multiple color and depth video coding using a hierarchical representation," IEEE Transactions on Circuits and Systems for Video Technology, vol. 17, no. 11, pp. 1450-1460, 2007.

[3] P. Merkle, A. Smolic, K. Müller, and T. Wiegand, "Efficient prediction structures for multiview video coding," IEEE Transactions on Circuits and Systems for Video Technology, vol. 17, no. 11, pp. 1461-1473, 2007.

[4] M. Flierl, A. Mavlankar, and B. Girod, "Motion and disparity compensated coding for multiview video," IEEE Transactions on Circuits and Systems for Video Technology, vol. 17, no. 11, pp. 1474-1484, 2007.

[5] S. Shimizu, M. Kitahara, H. Kimata, K. Kamikura, and Y. Yashima, "View scalable multiview video coding using 3D warping with depth map," IEEE Transactions on Circuits and Systems for Video Technology, vol. 17, no. 11, pp. 1485-1495, 2007.

[6] X. San, H. Cai, J.-G. Lou, and J. Li, "Multiview image coding based on geometric prediction," IEEE Transactions on Circuits and Systems for Video Technology, vol. 17, no. 11, pp. 15361548, 2007.

[7] A. Smolic, K. Mueller, N. Stefanoski, et al., "Coding algorithms for 3DTV-a survey," IEEE Transactions on Circuits and Systems for Video Technology, vol. 17, no. 11, pp. 1606-1621, 2007.

[8] ISO/IEC JTC 1/SC 29/WG 11, "Committee draft of ISO/IEC 23002-3 auxiliary video data representations," WG 11 Doc. N8038. Montreux, Switzerland, April 2006.

[9] C. Fehn, "A 3D-TV system based on video plus depth information," in Proceedings of the 37th Asilomar Conference on Signals, Systems and Computers, vol. 2, pp. 1529-1533, Pacific Grove, Calif, USA, November 2003.

[10] S. Grewatsch and E. Müller, "Sharing of motion vectors in 3D video coding," in Proceedings of IEEE International Conference on Image Processing (ICIP '04), vol. 5, pp. 32713274, Singapore, October 2004.

[11] H. Oh and Y.-S. Ho, "H.264-based depth map sequence coding using motion information of corresponding texture video," in Proceedings of the 1st Pacific Rim Symposium on Advances in Image and Video Technology (PSIVT '06), pp. 898-907, Hsinchu, Taiwan, December 2006.

[12] C. Fehn, "Depth-image-based rendering (DIBR), compression and transmission for a new approach on 3D-TV," in Stereoscopic Displays and Virtual Reality Systems XI, vol. 5291 of Proceedings of SPIE, pp. 93-104, San Jose, Calif, USA, January 2004.

[13] R. I. Hartley and A. Zisserman, Multiple View Geometry in Computer Vision, Cambridge University Press, Cambridge, UK, 2nd edition, 2004.

[14] L. B. Stelmach, W. J. Tam, D. V. Meegan, A. Vincent, and P. Corriveau, "Human perception of mismatched stereoscopic 3D inputs," in Proceedings of IEEE International Conference on Image Processing (ICIP '00), vol. 1, pp. 5-8, Vancouver, Canada, September 2000.

[15] P. Seuntiens, L. Meesters, and W. Ijsselsteijn, "Perceived quality of compressed stereoscopic images: effects of symmetric and asymmetric JPEG coding and camera separation," ACM
Transactions on Applied Perception, vol. 3, no. 2, pp. 95-109, 2006.

[16] I. Daribo, C. Tillier, and B. Pesquet-Popescu, "Distance dependent depth filtering in 3D warping for 3DTV," in Proceedings of IEEE International Workshop on Multimedia Signal Processing (MMSP '07), pp. 312-315, Crete, Greece, October 2007.

[17] W. R. Mark, L. McMillan, and G. Bishop, "Post-rendering 3D warping," in Proceedings of the Symposium on Interactive 3D Graphics, pp. 7-16, Providence, RI, USA, April 1997.

[18] M. Nalasani and W. D. Pan, "Performance evaluation of MPEG-2 codec with accurate motion estimation," in Proceedings of the 37th Annual Southeastern Symposium on System Theory (SSST '05), pp. 287-291, Tuskegee, Ala, USA, March 2005.

[19] G. J. Sullivan and T. Wiegand, "Rate-distortion optimization for: video compression," IEEE Signal Processing Magazine, vol. 15, no. 6, pp. 74-90, 1998.

[20] "Sequence microsoft ballet and breakdancers," 2004 http://research.microsoft.com/IVM/3DVideoDownload/.

[21] C. Fehn, K. Schüür, I. Feldmann, P. Kauff, and A. Smolic, "Distribution of ATTEST test sequences for EE4 in MPEG 3DAV," in MPEG Meeting - ISO/IEC JTC1/SC29/WG11, MPEG02/M9219, Awaji Island, Japan, December 2002.

[22] Digital bitrate, http://www.digitalbitrate.com/.

[23] ITU, "Methodology for the subjective assessment of the quality of television pictures," ITU-R Recommendation BT.500-10 (1974-2002), http://www.itu.int/. 